\title{
Esfínter artificial para la reconstrucción anorrectal total. Reporte preliminar y revisión de técnica quirúrgica*
}

\author{
Drs. MISAEL OCARES U. ${ }^{1}$, GINO CASELLI M. ${ }^{1}$, Int. BRUNO CASELLI M. ${ }^{2}$, \\ CLAUDIO BENAVIDES Y. ${ }^{1}$, LAURA FLORES O. ${ }^{1}$
}

Servicio de Cirugía Hospital Clínico Regional de Concepción.

2 Interno Facultad de Medicina.

Concepción, Chile.

\begin{abstract}
Artificial bowel sphincter for anorectal reconstruction. Preliminary report and review of surgical technique
\end{abstract}

Introduction: The artificial sphincter (ABS) has been proposed as an option for the treatment for severe refractory fecal incontinence. We have witnessed a great discussion on systematic reviews regarding the short-and long-term results of ABS, assessing its real benefit. Objective: To analyze the surgical outcomes of patients undergoing artificial sphincter implantation and review of surgical technique. Material and Methods: Between 2003 and 2007, the ABS system was introduced in four patients. The mean age was 34 years (13 to 54 years). Two patients were admitted for rectal agenesis, a severe anorectal trauma and abdominoperineal resection for a low rectal cancer. Results: Follow-up time was 12 to 60 months. We performed the explantation of the device for perineal wound infection in one case. All patients had mild incontinence ABS showed a decrease an average of 3.5 points to 12 months of follow-up on the Wexner Scale. Conclusions: The installation of the artificial sphincter is a feasible and safe alternative for anorectal reconstruction in selected patients. The morbidity and explantation of the series is similar to other international reports.

Key words: Artificial bowel sphincter, severe faecal incontinence, total anorectal reconstruction, rectal agenesis.

\section{Resumen}

Introducción: El esfínter artificial (siglas en inglés ABS) ha sido propuesto como una opción para el tratamiento de incontinencia fecal severa refractaria. Hemos sido testigos de una gran discusión en revisiones sistemáticas respecto a los resultados a corto y largo plazo del ABS, evaluando su real beneficio. Objetivos: Analizar los resultados quirúrgicos de los pacientes sometidos a implante de esfínter artificial y revisar la técnica quirúrgica. Material y Métodos: Entre los años 2003 y 2007 se implantó el sistema ABS en cuatro pacientes. La edad media fue de 34 años (13 a 54 años). Dos pacientes ingresaron por agenesia rectal, uno por trauma anorrectal severo y otro por resección abdominoperineal de un cáncer de recto bajo.

*Recibido el 21 de Octubre de 2008 y aceptado para publicación el 3 de Marzo de 2009.

Correspondencia: Dr. Misael Ocares U.

Chacabuco esq. Janequeo. 4to Piso, Concepción, Chile.

E-mail: misaelocares@udec.cl 
Resultados: El tiempo de seguimiento fue de 12 a 60 meses. Se realizó la explantación del dispositivo por infección de herida perineal en un caso. Todos los pacientes que mantuvieron el ABS presentaron incontinencia leve con un descenso a los 12 meses de seguimiento a una media de 3,5 puntos en la escala de Wexner. Conclusiones: La instalación del esfinter artificial es una alternativa segura y realizable para la reconstrucción anorrectal en pacientes seleccionados. El porcentaje de morbilidad y explantación de la serie es similar a otros reportes internacionales.

Palabras clave: Esfínter anal artificial, incontinencia fecal severa, reconstrucción anorrectal total, agenesia rectal.

\section{Introducción}

En Chile, la prevalencia estimada de incontinencia fecal para la población en centros de salud es de $2,7 \%{ }^{1}$. En casas de reposo ésta asciende a un $45 \%$, constituyéndose en una condición social y personal incapacitante ${ }^{2}$. Dependiendo de la severidad, pueden ser utilizados los medicamentos antidiarreicos, el biofeedback o la neuromodulación; llegando hasta la cirugía, que incluye reparación esfinteriana, graciloplastía e implante de esfínter artificial. La falla de todas estas alternativas resulta en la consideración de una colostomía ${ }^{3,4}$, representando una condición definitiva que afecta enormemente la calidad de vida ${ }^{5}$. Reportes recientes indican que la instalación de un esfínter artificial es razonablemente exitosa en casos seleccionados de reconstrucción anorrectal ${ }^{6}$. Sin embargo, el riesgo de infección y erosión del sistema alrededor del canal anal se mantiene como un problema de difícil manejo. No hay estudios metodológicamente óptimos que comprueben el real beneficio de éste en cirugía reconstructiva anorrectal. Este artículo muestra la experiencia inicial de nuestro grupo en la instalación de esfínter artificial para la reconstrucción anorrectal.

\section{Material y Método}

Entre los años 2003 y 2007 se realizó el implante de esfínter anal artificial a cuatro pacientes. La primera paciente era portadora de una lesión severa del anorrecto de etiología no obstétrica. Dos pacientes eran portadores de una malformación anorrectal congénita (agenesia rectal) y el último paciente se sometió a una resección abdominoperineal por un tumor de recto bajo. Todos los pacientes se sometieron a evaluación sicológica previo a la cirugía. Además se les explicó la técnica y se obtuvo el consentimiento informado. El esfínter artificial implantado A.M.I ${ }^{\mathrm{TM}}$ Soft Anal Band System (Agency for Medical Innovations, Feldkirch, Austria) fue el mismo para los cuatro pacientes de la serie (Figura 1). En el paciente sometido a reconstrucción ano- rrectal total (RAT) posterior a una resección abdominoperineal, fue necesario una amplia movilización del ángulo esplénico para alojar el colon a nivel perineal, siendo dificultosa esta parte de la cirugía dada las condiciones intraabdominales anormales por laparotomía previa. En todos los pacientes fue necesario realizar una ileostomía de protección en asa hasta lograr la cicatrización completa de las heridas perineales. El grado de continencia fue medido de acuerdo con la Escala de Continencia de Wexner $^{4}(\mathrm{EW})$, cuyo rango va de 0 en caso de una continencia normal a 20 para incontinencia total. $\mathrm{La}$ constipación severa fue medida con la Escala de Wexner para constipación ${ }^{7}$ (EC), cuyo rango va de 0 para evacuación normal y 30 para máxima constipación.

\section{Técnica quirúrgica}

La instalación del esfínter anal artificial ha sido descrito $^{8}$ y corresponde a una técnica estandarizada:

a) Se realizó preparación de colon anterógrada con solución de polietilenglicol asociado a lavado retrógrado de colon con enemas evacuantes.

b) El paciente se puso en posición de litotomía modificada, debiendo permitir un abordaje abdominal y perineal.

c) Se procuró poner especial atención a la asepsia a través de todo el procedimiento. Esta incluyó lavado preoperatorio antiséptico, además de la exclusión de la región perineal y canal anal del campo operatorio. Además se realizó lavado constante de los abordajes quirúrgicos con solución fisiológica y gentamicina.

d) Se usó antibióticoprofilaxis con Ceftriaxona 1 gr iv y Metronidazol $500 \mathrm{mg}$ iv 1 hora preoperatoria. Transcurridas dos horas desde el inicio de la cirugía se repitió la misma dosis de la cefalosporina utilizada.

e) En el periné se realizó incisiones transversales en frente del ano y no verticales bilaterales. Se utilizó esta incisión ya que otorga una mejor exposición desde una disección anterior. El largo del cuff oclusivo se determina usando un medidor especialmente diseñado, debiendo medir $1 \mathrm{~cm}$ más que 


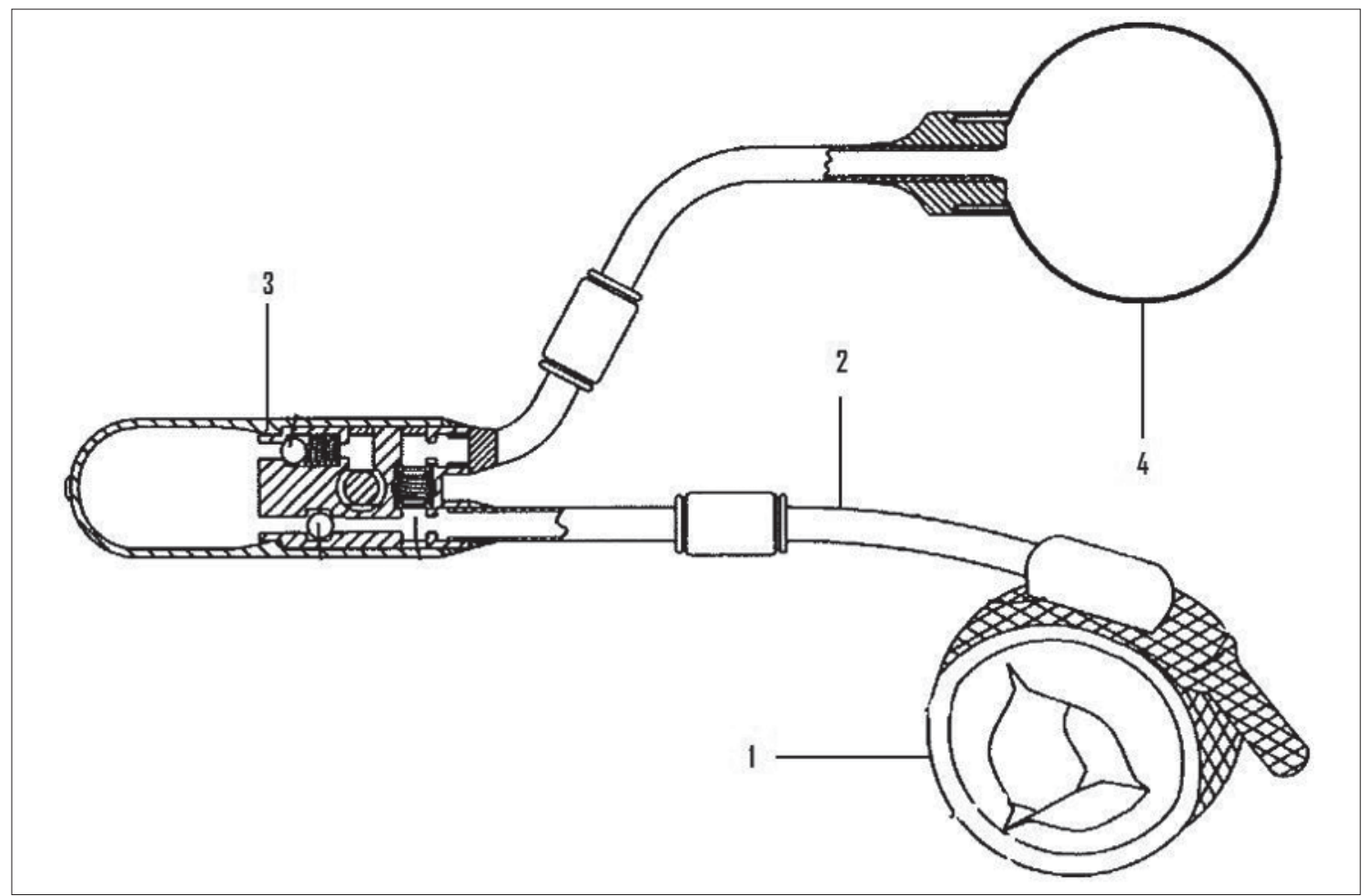

Figura 1. Esfínter anal artificial. Componentes del sistema ABS: 1) Cuff perianal; 2) Tubo siliconado; 3) Balón de presión; 4) Bomba de control.

la dimensión del cuff para la circunferencia del esfinter.

f) El cuff perianal es el primer componente del sistema a instalar (Figura 1-1). Posteriormente se debe tunelizar por el tejido subcutáneo el tubo siliconado (Figura 1-2) desde el neoesfínter hacia la incisión abdominal con una aguja atraumática. Luego se crea un bolsillo para alojar el balón de presión o activador (Figura 1-3). El cuff es primero presurizado por conexión con el balón, el cual es llenado con $55 \mathrm{cc}$ de líquido radioópaco. La cantidad de fluido mantenido en el cuff posterior a la presurización es cuidadosamente medido cuando se vacía el balón; usualmente entre 4 y 8 cc. El balón se implanta vacío, posteriormente llenándose con 40 cc de líquido radioópaco, volumen al cual la presión otorgada al cuff corresponde a los valores determinados por el manufacturador (entre 80 y 100 $\mathrm{cm}$ de agua). Posteriormente la aponeurosis es cuidadosamente cerrada.

g) Luego la bomba de control es posicionada (Figura 1-4). Al ser este el único componente del sistema que el paciente debe sentir y manipular, se debe acceder fácilmente. En dos de los cuatro casos la instalación del balón regulador de presión se realizó extraperitonealmente en la fosa iliaca izquierda. Se prefiere este sitio por su facilidad de disección y escasa vascularización. En el paciente con resección abdominoperineal con colostomía terminal se prefirió ubicar el activador en fosa ilíaca derecha. En la paciente con trauma perineal se decidió instalar el balón en el labio mayor. Posterior a esto, el cuff oclusivo y el balón regulador deben conectarse a la bomba. Finalmente, la piel se cierra con puntos separados de material no reabsorbible (Figura 2).

h) La activación del ABS se debe realizar inicialmente a las ocho semanas después de la instalación.

\section{Resultados}

La edad media fue de 34,5 años. El tiempo operatorio fue de $140 \pm 40 \mathrm{~min}(100-180 \mathrm{~min})$. La media de seguimiento fue de 13,5 meses (12 a 60 meses). Tres de los pacientes presentaron complicaciones postoperatorias (infección e impactación fecal) con un caso de explantación del sistema ABS. Además un paciente presenta dolor crónico en la región perianal. 


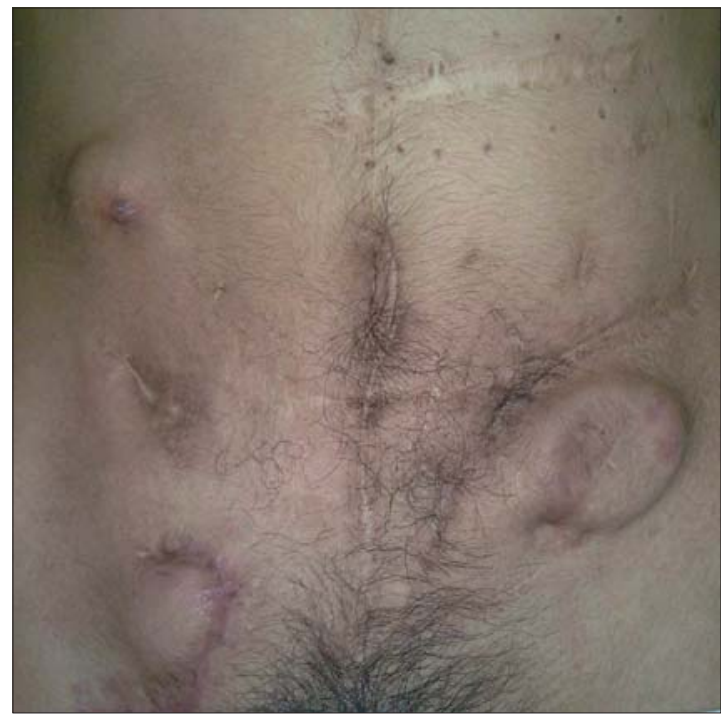

Figura 2. Aspecto final.

\section{Caso 1}

Mujer de 54 años. Ingresada con diagnóstico de trauma anorrectal severo no iatrogénico con una incontinencia fecal severa (EW: 16). Evolucionó en el postoperatorio sin problemas, logrando además una mejoría en la continencia, representada con una EW que descendió a 6 y 3 en seis y doce meses de control, respectivamente. Ha presentado 2 episodios de constipación (EC: 15) con un episodio de impactación fecal a los 2 años.

\section{Caso 2}

Hombre de 14 años. Portador de agenesia anorrectal. Se objetivó una EW de 18 durante el preoperatorio. Durante el control a los 12 meses presenta una mejoría importante de su continencia, evidenciando incontinencia leve a gases (EW: 3). Ingresó a nuestro centro 2 meses después de su cirugía por un cuadro de impactación fecal (EC: 18), el cual se manejó médicamente. Desarrolló, 6 meses después de la implantación, la exposición de la válvula esfintérica, debiéndose tallar un nuevo bolsillo cutáneo, evolucionando sin incidentes. Actualmente presenta dolor anal, el cual se incrementa al inflar la válvula para lograr continencia total, debiendo manejar presiones de inflado más bajas.

\section{Caso 3}

Hombre de 13 años. Portador de agenesia rectal. Ha mejorado su continencia de base (EW: 18) a 7 y 4 en controles postoperatorios (6 y 12 meses plazo). No ha presentado complicaciones.

\section{Caso 4}

Hombre de 53 años. Sometido a RAT 24 meses después de una resección abdominoperineal por un adenocarcinoma de recto bajo con compromiso esfinteriano. Manejado con terapia neoadyuvante. Se realizó colostomía perineal e instalación de ABS, ingresando 4 meses después con infección y granulomas en sitio de inserción del activador y balón de presión. Por infección y exposición de la válvula perineal se decide realizar explantación del ABS.

\section{Discusión}

La efectividad del esfinter artificial, así como sus indicaciones, ha sido revisada en muchos artícu$\operatorname{los}^{9,10}$, disponiéndose sólo de publicaciones de series limitadas ${ }^{11}$. Así se ha determinado la mejor efectividad del ABS para la incontinencia fecal severa, siendo escasos los artículos referentes a otras indicaciones menos frecuentes de ABS (Tabla 1).

Nuestra serie reporta una morbilidad en 3 de 4 pacientes. Se presentó un episodio de impactación fecal en 2 pacientes e infección de herida de implante en otros 2 casos, uno de los cuales terminó con la explantación del sistema. El porcentaje de explantación de $25 \%$ concuerda con comunicaciones internacionales ${ }^{8}$. Además, destacamos que uno de nuestros pacientes ha presentado dolor crónico que se ha manejado con analgésicos, con un EVA de 3 al momento del control. La morbilidad ha sido un tema recurrente en las revisiones acerca de la seguridad del implante. Dentro de ellas se ha incluido la infección postoperatoria, la impactación fecal, dolor crónico y la erosión rectal. Series de casos han informado tasas de morbilidad tan altas

Tabla 1. Indicaciones y contraindicaciones de esfínter artificial

\begin{tabular}{|c|c|}
\hline Tipo de indicación & Diagnóstico clínico \\
\hline Indicaciones & $\begin{array}{l}\text { Trauma esfinteriano } \\
\text { Incontinencia neurogénica } \\
\text { (idiopática) } \\
\text { Incontinencia neurológica }\end{array}$ \\
\hline Indicaciones relativas & $\begin{array}{l}\text { Ano imperforado } \\
\text { Trauma perineal severo } \\
\text { Senilidad, Diabetes }\end{array}$ \\
\hline Contraindicaciones & $\begin{array}{l}\text { Descenso perineal severo } \\
\text { Constipación severa } \\
\text { Irradiación perineal } \\
\text { Sepsis perineal, E. Crohn, } \\
\text { Sexo anal }\end{array}$ \\
\hline
\end{tabular}


como $100 \%{ }^{12}$, con porcentajes de explantación cercanos al $30 \%$ en los estudios de mejor calidad metodológica. Sin embargo, la instalación del esfínter artificial parece ser segura. No existen reportes de mortalidad asociada a esta cirugía. Las complicaciones ligadas al ABS están localizadas en especial en la región anorrectal y no están asociadas a ninguna enfermedad sistémica.

Sólo un selecto grupo de patologías han hecho necesario el uso de ABS para lograr la restitución del complejo anorrectal y lograr continencia (malformaciones anorrectales, cirugía reconstructiva oncológica y trauma anorrectal). En casos de incontinencia por agenesia rectal existe un bajo porcentaje de éxito ${ }^{13}$. Esta malformación se acompaña de ausencia de sensibilidad anal y de reservorio rectal, además de la existencia de desórdenes motores colónicos asociados, que hacen que estas técnicas de reconstitución esfinteriana tengan resultados inciertos, no existiendo parámetros predictivos acerca del éxito de ABS bajo esta indicación ${ }^{13}$. Nuestra serie presenta dos casos exitosos de RAT con un seguimiento de 12 meses a pacientes portadores de agenesia rectal. Durante el tiempo de seguimiento de ambos pacientes, uno de ellos ha presentado episodios de impactación fecal.

Respecto a la reconstrucción anorrectal posterior a una resección abdominoperineal, Romano et $\mathrm{al}^{14}$, reporta ocho casos de implantación posteriores a una operación de Miles, incluyendo un caso de cirugía sincrónica. Cabe destacar que pacientes con tumores rectales con etapificación preoperatoria $\mathrm{T}_{1-2} \mathrm{~N}_{0}$ son los mejores candidatos para reconstrucción sincrónica ${ }^{14}$, aunque en casos de tumores más avanzados, como en esta serie, preferimos esperar un período de 2 años libre de enfermedad para realizar la reconstrucción. Estos casos deben estar precedidos de un buen seguimiento oncológico ${ }^{13}$. En nuestra limitada experiencia, la implantación del esfínter artificial alrededor de la colostomía perineal posterior a una reconstrucción por cáncer parece ser una cirugía segura. Es probable que la radioterapia externa haya sido factor para que la infección perineal hiciera fracasar nuestro intento.

Los traumas de la región anorrectal son raros, aunque las injurias iatrogénicas no son infrecuentes. El reconocimiento inmediato es vital para un buen pronóstico, pudiendo obviar la necesidad de una ostomía $^{15}$ optando por el uso del esfínter artificial. Destacamos que no hay estudios que describan la experiencia del uso de ABS en lesiones anorrectales traumáticas no obstétricas. En nuestra serie, sólo una paciente se sometió a implante de ABS por esta causa, con buenos resultados, pero con episodios aislados de impactación fecal.

Por último, consideramos que existen dos facto- res a tomar en cuenta al momento de realizar una RAT. Primero, la dificultad de lograr una comparación entre los scores pre y postoperatorios, ya que no se entregan datos objetivos para la comparación y segundo, la capacidad de manipular y controlar la bomba de presión, que necesita de una capacidad intelectual suficiente para comprender el funcionamiento del sistema y asegurar la evacuación rectal ${ }^{16}$

\section{Conclusiones}

Nuestros resultados corresponden a una experiencia inicial. El esfínter anal artificial es una opción válida para la reconstrucción, en pacientes seleccionados con malformaciones anorrectales, traumas perineales severos y resección abdominoperineal por neoplasia. Aunque es un procedimiento seguro y sin complicaciones graves, aún presenta porcentajes muy elevados de morbilidad. Además el costo del sistema permanece muy alto para la realidad nacional. Aún así, los resultados de esta cirugía nos alientan a seguir mientras no existan otros enfoques menos invasivos para el manejo de estas patologías, manteniendo un criterio de selección riguroso de los pacientes para obtener los mejores resultados.

\section{Referencias}

1. Zárate A, López F, Vergara F, Badilla N, Viviani P. Prevalencia de la incontinencia fecal en centros de salud y casas de reposo. Rev Méd Chile 2004; 136 : 867-872.

2. Lynch A, Dobbs B, Keating J, Frizelle F. The prevalence of faecal incontinence and constipation in a general New Zealand population; a postal survey. NZ Med J 2001; 114: 474-477.

3. Kamm M. Faecal incontinence: common and treatable. Med J Aust 2002; 176: 47-48.

4. Jorge J, Wexner S. Etiology and management of fecal incontinence. Dis Colon Rectum 1993; 36: 77-97.

5. Nugent K, Daniels P, Stewart B, Patankar R, Johnson C. Quality of life in stoma patients. Dis Colon Rectum 1999; 42: 1569-1574.

6. Gurusamy K, Marzouk D, Benzinger H. A review of contemporary surgical alternatives to permanente colostomy. Int J Surg 2005; 3: 193-205.

7. Agachan F, Chen T, Pfeifer J, Reissman P, Wexner S. A constipation scoring system to simplify evaluation and management of constipated patients. Dis Colon Rectum 1996; 39: 681-685.

8. O'Brien P, Skinner S. Restoring control: the Acticon Neosphincter artificial bowel sphincter in the 
treatment of anal incontinence. Dis Colon Rectum 2000; 43: 1213-1216.

9. Vaizey C, Kamm M, Gold D, Bartram C, Halligan S, NIcholls R. Clinical, physiological and radiological study of a new purpose-designed artificial bowel sphincter. Lancet 1998; 352: 105-109.

10. Vaizey C, Kamm M, Nicholls R. Recent advances in the surgical treatment of faecal incontinence. $\mathrm{Br} \mathrm{J}$ Surg 1998; 85: 596-603.

11. Mundy L, Merlin T, Maddern G, Hiller J. Systematic review of safety and effectiveness of an artificial bowel sphincter for faecal incontinente. Br J Surg 2004; 91: 665-672.

12. Lehur P, Michot F, Denis P, Grise P, Leborge J, Teniere $\mathrm{P}$, et al. Results of artificial sphincter in severe anal incontinence. Report of 14 consecutive implantations. Dis Colon Rectum 1996; 39: 13521355.

13. Romano G, LA Torre F, Cutini G, Bianco F, Esposito P. Total anorectal reconstruction with an artificial bowel sphincter. Report of five cases with minimum follow-up of 6 months. Colorectal Dis 2002; 4: 339344.

14. Romano G, LA Torre F, Cutini G, Bianco F, Esposito $\mathrm{P}$, Montori A. Total anorectal reconstruction with the artificial bowel sphincter: Report of eight cases. Dis Colon Rectum 2003; 46: 730-734.

15. Helliger M. Anal trauma and foreign bodies. Surg Clin North Am 2002; 82: 1253-1260.

16. Hoch J, Skaba R, Jech Z. Artificial sphincter in patients with congenital anorectal malformation. Rozhl Chir 2007; 86: 170-173. 